\title{
Determination of Tranexamic Acid and Vitamins by High Performance Liquid Chromatography with Peroxyoxalate Chemiluminescence Detection
}

\author{
Kenji Sato, Yuichi Tobita, Keiko Iwabuchr and Shigeyuki TaNaka \\ Department of Fundamental Science, College of Science and Engineering, Iwaki Meisei University, \\ Iwaki, Fukushima 970, Japan
}

\begin{abstract}
A high performance liquid chromatographic determination of tranexamic acid and two vitamins, i.e., vitamin $U$ chloride and anthranilic acid, with chemiluminescence detection was investigated. o-Phthalaldehyde(OPA) solution which contained 2-mercaptoethanol was used as a fluorescent labeling reagent. OPA solution was prepared by mixing OPA and 2-mercaptoethanol in ethanol, and then diluting with borate buffer solution. Bis(2,4,6-trichlorophenyl)oxalate and hydrogen peroxide were used a postcolumn chemiluminescent reagent. The intensity of the chemiluminescence was in the order of tranexamic acid $>$ vitamin $U$ chloride $>a n t h r a n i l i c$ acid. The calibration curves for the compounds were linear at least in the range from $1 \times 10^{-7} \mathrm{M}$ to $1 \times 10^{-4} \mathrm{M}$. The detection limits for tranexamic acid, vitamin $U$ chloride and anthranilic acid were $5 \times 10^{-8} \mathrm{M}, 4 \times 10^{-7} \mathrm{M}$ and $7 \times 10^{-7} \mathrm{M}$, respectively. The relative standard deviation was less than $9 \%(n=5)$. The proposed method was applied to the determination of tranexamic acid and vitamin $U$ chloride in commercially available medicine.
\end{abstract}

Key words Chemiluminescence, tranexamic acid, anthranilic acid, vitamin U chloride, high performance liquid chromatography, peroxyoxalate reagent

Chemiluminescence(CL) assays have been extensively investigated because of their such promising properties as high sensitivity, extensive dynamic range, and generally inexpensive instrumentation. Among the $\mathrm{CL}$ reactions, peroxyoxalate $\mathrm{CL}$ reaction was applied to the determination of metal ions $\mathbf{s}^{1}$, environmental pollution materials ${ }^{2}$ and biogenic substances. ${ }^{3 \cdot 5}$ For the analyses of these compounds, the combination of high performance liquid chromatography(HPLC) and $C L$ detection was often used. If the analytes were non-fluorescent, fluorogenic reactions could increase the utility of this detection mode. Phenylisothiocyanate, $o$-phthalaldehyde(OPA) ${ }^{7}$ and dansyl chloride $e^{8}$ were the most popular reagents for pre-column fluorescence derivatization. OPA was well known to react with primary amines or primary amino acids to give the corresponding fluorophores. In this work, OPA was selected from the reason that it could allow an easy operation at room temperature. Fluorogenic reaction with $\mathrm{OPA}$ and $\mathrm{CL}$ reactions involved in peroxyoxalate $\mathrm{CL}$ is illustrated briefly in Fig. 1.

In Fig. 1(1), an analyte, that is, primary amino compound, was allowed to react with OPA to afford highly fluorescent isoindole derivative $(F)$ in the presence of 2-mercaptoethanol as a reducing

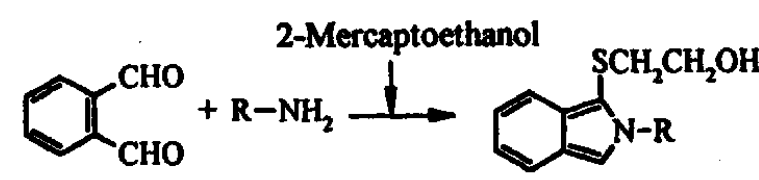

o-Phthalaldehyde Isoindole derivative (F)<smiles>O=C(O[Al])C(=O)O[Al]</smiles>

1,2-Dioxetanedione

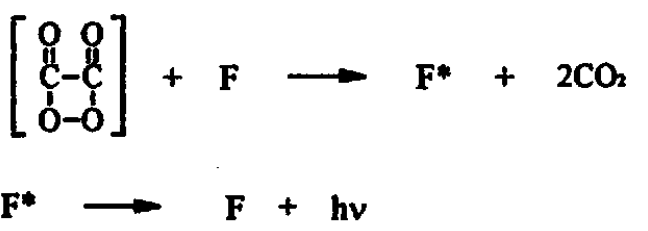

Fig. 1 Fluorogenic reaction and peroxyolalate CL reaction.

reagent. Whereas, as shown in Fig. 1(2), the reaction of an aryl oxalate ester and hydrogen peroxide forms 


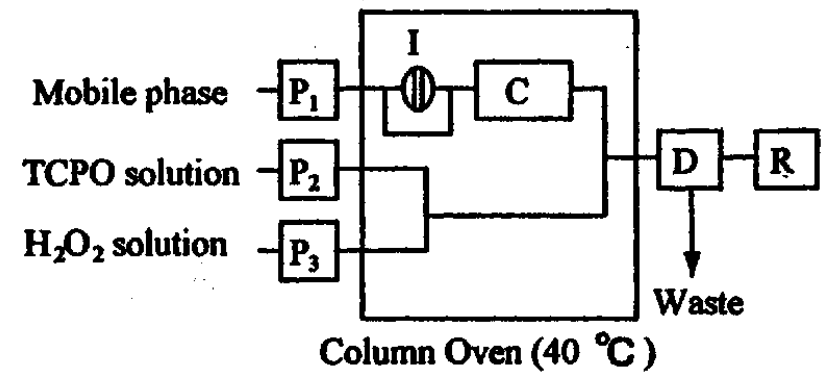

Fig. 2 Schematic diagram of the HPLC with CL detection. $P_{1}-P_{3}$, pump; $I$, injector; $C$, column; $\mathrm{D}$, detector; $\mathrm{R}$, recorder.

1,2-dioxetanedione(intermediate) and this product can excite fluorescer(F, Fig. 1(3)). The excited fluorescent compound $\left(\mathrm{F}^{*}\right)$ returns to the ground state with the emission of light(Fig. 1(4)). An intensity of the light emitted, that is, CL intensity, is dependent on the concentration of $F$.

In this study, we investigated a peroxyoxalate $\mathrm{CL}$ method for the determination of tranexamic acid, vitamin $U$ chloride and anthranilic acid, based on the conversion of these compounds to fluorescent isoindole derivatives with OPA. The fluorogenic reaction proceeds as shown in Fig. 1(1), because these compounds contain a primary amine functional group. The reversed-phase HPLC which was equipped with a CL detector was used. Bis(2,4,6-trichlorophenyl)oxalate(TCPO) and hydrogen peroxide $\left(\mathrm{H}_{2} \mathrm{O}_{2}\right)$ were used a postcolumn chemiluminescent reagent. The proposed method was applied to the determination of tranexamic acid and vitamin $U$ chloride in commercially available medicine.

\section{Experimental}

\section{Apparatus}

Figure 2 illustrates the HPLC system used with CL detection. The high performance liquid chromatograph consisted of three pumps(JASCO, model 880-PU and PU-980), a Rheodyn 7125 injector, a CL detector(JASCO, model 825-CL) and a recorder(RIKADENKI, model R-62). Three pumps were used for delivering the mobile phase, TCPO and hydrogen peroxide solution. The sample injector was equipped with a $20-\mu l$ injection loop. The analytical column used was a LiChrosorb RP-18-5 (250 x $4.6 \mathrm{~mm}$ i.d.). The column temperature was $40^{\circ} \mathrm{C}$. The flow rates of mobile phase, TCPO solution and hydrogen peroxide solution were $1.0,2.0$ and $2.5 \mathrm{ml} / \mathrm{min}$.

\section{Reagents}

OPA and 2-mercaptoethanol were purchased from Tokyo Kasei(Tokyo, Japan) and Kanto Chemicals (Tokyo, Japan), respectively. The fluorogenic reagent (referred to this reagent as OPA solution) was prepared by mixing $200 \mu \mathrm{l}$ of OPA $(10 \mathrm{mg} / \mathrm{ml}$ in

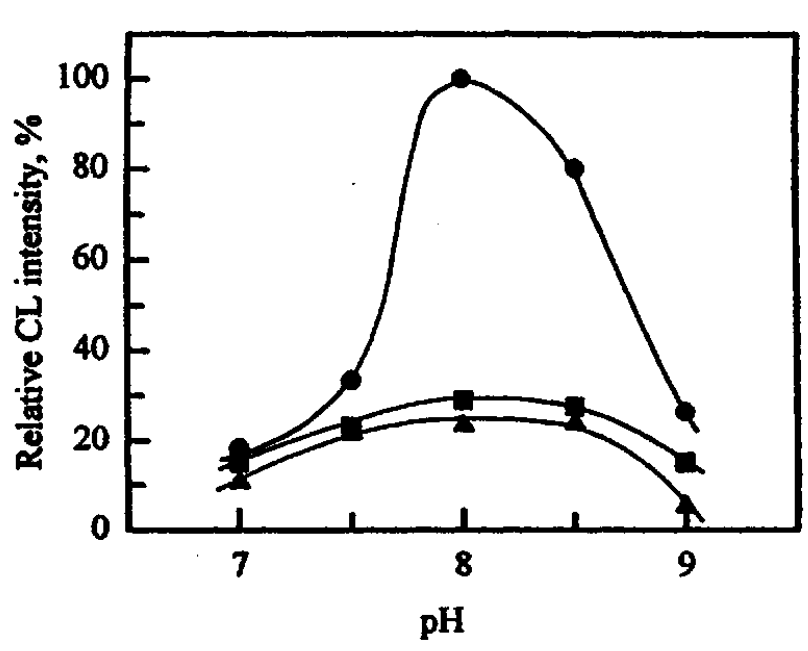

Fig. 3 Effect of $\mathrm{pH}$ on the $\mathrm{CL}$ intensity. $\bullet, 1 \times 10^{-5} \mathrm{M}$ tranexamic acid ; $\triangle, 1 \times 10^{-5} \mathrm{M}$ vitamin $U$ chloride ; $\varpi, 1 \times 10^{-5} \mathrm{M}$ anthranilic acid.

ethanol) and $100 \mu$ l of 2-mercaptoethanol $(5 \mu \mathrm{l} / \mathrm{ml}$ in ethanol) and diluting to $20 \mathrm{ml}$ with $0.05 \mathrm{M}$ borate buffer solution of $\mathrm{pH} 8$. Bis(2,4,6-trichlorophenyl) oxalate(TCPO) was purchased from Wako Pure Chemicals(Osaka, Japan). A $0.5 \mathrm{mM}$ TCPO was dissolved in acetonitrile containing ethyl acetate at $10 \%(\mathrm{v} / \mathrm{v})$ concentration. Hydrogen peroxide was obtained from Mitsubishi Gas Kagaku(Tokyo, Japan). A $5 \mathrm{mM}$ hydrogen peroxide solution was prepared by diluting with acetonitrile containing $10 \%(\mathrm{v} / \mathrm{v})$ imidazole buffer(pH 6.5). The mobile phase was $20 \%(v / v)$ acetonitrile aqueous solution containing $10 \%(\mathrm{v} / \mathrm{v})$ ethanol. Tranexamic acid (trans- 4 -aminomethyl- 1 -cyclohexanecarboxylic acid) and anthranilic acid were purchased form Wako Pure Chemicals. Vitamin U chloride was obtained from Tokyo Kasei. All other chemicals were of analytical-reagent grade and were used without further purification.

\section{Procedure}

To assay tranexamic acid, vitamin $U$ chloride and anthranilic acid in aqueous solution, a $100 \mu \mathrm{l}$ of the standard solution(tranexamic acid and vitamin $U$ chloride: $1 \times 10^{-5} \mathrm{M}$, anthranilic acid: $1 \times 10^{-4} \mathrm{M}$ ) was mixed with $800 \mu$ l of OPA solution in a glass tube. The CL intensity was measured by injecting the mixed solution immediately. The measurement of a blank without analyte was done in the same way as standard solution.

\section{Results and discussion}

\section{Optimization of fluorescent labeling}

In order to decide the optimum operating parameters for the HPLC determination of tranexamic acid, vitamin $U$ chloride and anthranilic acid, preliminary investigations were conducted by removing an analytical column. The most characteristic factors that affect the fluorogenic 
reaction with OPA solution were $\mathrm{pH}, \mathrm{OPA}$ and 2mercaptoethanol concentration, and reaction time of the fluorogenic reaction, and so on. Firstly, the optimum $\mathrm{pH}$ region for the fluorogenic reaction was investigated. The results obtained were plotted in Fig. 3. Tranexamic acid exhibited the highest CL intensity compared with the other compounds at all $\mathrm{pH}$ region. The $\mathrm{CL}$ intensity of tranexamic acid in the range of the neighborhood of $\mathrm{pH} 8$ was greater than that in the other $\mathrm{pH}$ value. Whereas, vitamin $U$ chloride and anthranilic acid showed approximately constant $\mathrm{CL}$ intensity in the range of pH 8 - 8.5. Therefore, pH 8 was adopted for the reaction of the fluorescence derivatization.

At room temperature, the fluorogenic reaction proceeds readily. After the OPA solution was added, the maximum $\mathrm{CL}$ intensity was observed in $2 \mathrm{~min}$, then the $C L$ intensity decreased slowly with the progress of the reaction time. This behavior was probably due to the decomposition of OPAderivative compound. In this work, therefore, the sample solution was injected immediately after the compound was mixed with OPA solution. The influences of the concentrations of OPA and 2mercaptoethanol on the $\mathrm{CL}$ intensity were investigated. The maximum and constant $\mathrm{CL}$ intensities were obtained at the concentrations of OPA and 2-mercaptoethanol higher than about $2 \times$ $10^{-3} \mathrm{M}$ and $1.8 \times 10^{-4} \mathrm{M}$, respectively. The relative $\mathrm{CL}$ intensity ratio between sample solution and blank was calculated. As a result, $7.5 \times 10^{-4} \mathrm{M}$ and $3.5 \mathrm{x}$ $10^{-4} \mathrm{M}$ were selected respectively as the concentrations of OPA and 2-mercaptoethanol.

\section{Effect of organic solvent on $C L$ intensity}

The various organic solvents such as ethanol, methanol, acetonitrile and acetone were added to the mobile phase to enhance CL intensity. The CL intensity was measured by using HPLC equipped with an analytical column. The highest CL intensity was obtained by adding ethanol. In addition, the CL intensity was measured by using the various mobile phases which contained ethanol from $5 \%(v / v)$ to $20 \%(v / v)$. Although the CL intensity increased with

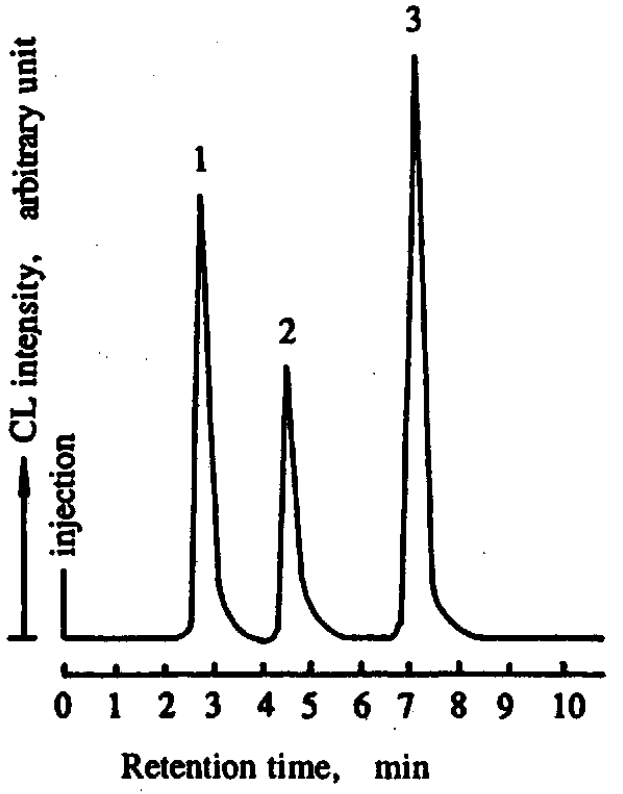

Fig. 4 Typical chromatogram of OPA-derivatives of tranexamic acid, vitamin $U$ chloride and anthranilic acid. $1,1 \times 10^{-3} \mathrm{M}$ anthranilic acid; $2,2 \times 10^{-4} \mathrm{M}$ vitamin $\mathrm{U}$ chloride; $3,1 \times 10^{-4} \mathrm{M}$ tranexamic acid.

an increase ethanol concentration, the retention times of the compounds were decreased. Therefore, $20 \%(v / v)$ acetonitrile aqueous solution which contained $10 \%(\mathrm{v} / \mathrm{v})$ ethanol was adopted as a mobile phase.

The typical chromatogram obtained was shown in Fig. 4. The retention times of tranexamic acid, vitamin $U$ chloride and anthranilic acid were about $2.9 \mathrm{~min}, 4.5 \mathrm{~min}$ and $7.5 \mathrm{~min}$, respectively. The peaks for the compounds were separated completely within $8.5 \mathrm{~min}$. The intensity of the $\mathrm{CL}$ was in the order of tranexamic acid> vitamin $U$ chloride >anthranilic acid.

\section{Calibration and detection limit}

The calibration curves for the compounds were

Table 1 Analytical results for determination of tranexamic acid

\begin{tabular}{|c|c|c|c|c|c|c|c|}
\hline \multirow[t]{2}{*}{ Sample } & \multirow{2}{*}{$\begin{array}{l}\text { Sample } \\
\text { taken/g }\end{array}$} & \multirow[t]{2}{*}{ Aliquot } & \multicolumn{3}{|c|}{ tranexamic acid } & \multirow{2}{*}{$\begin{array}{c}\text { Recovery } \\
1 \%\end{array}$} & \multirow{2}{*}{$\begin{array}{l}\text { Labeled } \\
\text { amount/ mg }\end{array}$} \\
\hline & & & added $\mu g$ & found $/ \mu g^{*}$ & in sample/ $\mathrm{mg}$ & & \\
\hline \multirow[t]{3}{*}{$\mathbf{A}$} & 0.2984 & $1 / 10000$ & 0 & 7.05 & 70.5 & & $70 / 0.3 \mathrm{~g}$ \\
\hline & & $1 / 10000$ & 5 & 12.2 & & 103 & \\
\hline & & $1 / 2000$ & 0 & 35.4 & 70.8 & & \\
\hline \multirow[t]{3}{*}{ B } & 0.3394 & $1 / 10000$ & 0 & 12.0 & 120 & & $125 / 0.3 \mathrm{~g}$ \\
\hline & & $1 / 10000$ & 5 & 16.8 & & 96.0 & \\
\hline & & $1 / 2000$ & 0 & 61.8 & 124 & & \\
\hline \multirow[t]{3}{*}{$\mathrm{C}$} & $1 \mathrm{ml}$ & $1 / 100$ & 0 & 4.98 & 0.498 & & \\
\hline & & $1 / 100$ & 5 & 10.1 & & 102 & \\
\hline & & $1 / 20$ & 0 & 24.7 & 0.494 & & \\
\hline
\end{tabular}

* Means of four experiments 
Table 2 Analytical results for determination of vitamin U chloride

\begin{tabular}{|c|c|c|c|c|c|c|c|}
\hline \multirow[t]{2}{*}{ Sample } & \multirow{2}{*}{$\begin{array}{l}\text { Sample } \\
\text { taken/g }\end{array}$} & \multirow[t]{2}{*}{ Aliquot } & \multicolumn{3}{|c|}{ vitamin $\mathrm{U}$ chloride } & \multirow{2}{*}{$\begin{array}{c}\text { Recovery } \\
1 \%\end{array}$} & \multirow{2}{*}{$\begin{array}{c}\text { Labeled } \\
\text { amount/mg }\end{array}$} \\
\hline & & & $\operatorname{addd} / \mu \mathrm{g}$ & found/ $\mu g^{*}$ & in sample/ $\mathrm{mg}$. & & \\
\hline \multirow[t]{3}{*}{$\bar{D}$} & 1.4743 & $1 / 5000$ & 0 & 10.1 & 50.5 & & $50 / 1.5 \mathrm{~g}$ \\
\hline & & $1 / 5000$ & 5 & 14.9 & & 96.0 & \\
\hline & & $1 / 1000$ & 0 & 50.0 & 50.0 & & \\
\hline \multirow[t]{3}{*}{ E } & 1.3032 & $1 / 5000$ & 0 & 9.68 & 48.4 & & $50 / 1.3 \mathrm{~g}$ \\
\hline & & $1 / 5000$ & 5 & 14.5 & & 96.4 & \\
\hline & & $1 / 1000$ & 0 & 49.6 & 49.6 & & \\
\hline \multirow[t]{3}{*}{$\mathrm{F}$} & 0.4446 & $1 / 2500$ & 0 & 10.1 & 25.3 & & $25 / 0.4 \mathrm{~g}$ \\
\hline & & $1 / 2500$ & 5 & 15.1 & & 100 & \\
\hline & & $1 / 500$ & 0 & 49.1 & 24.6 & & \\
\hline
\end{tabular}

* Means of four experiments

obtained by using proposed method under the optimized conditions. The relationship between chromatographic peak response(peak area) and concentration $\left(1 \times 10^{-7} \mathrm{M}-1 \times 10^{-4} \mathrm{M}\right)$ of the compounds was linear. The linear regression analysis resulted in the following equation: $Y=1.03 X$ +7.59 for tranexamic acid, $Y=1.10 X+7.22$ for vitamin $U$ chloride and $Y=1.05 X+6.48$ for anthranilic acid, where $Y=$ peak area $(\mu \mathrm{V}$ s) and $X$ $=$ concentration $(M)$. The regression coefficient was 0.999 . The detection limits (signal to noise ratio $=3$ ) for tranexamic acid, vitamin $U$ chloride and anthranilic acid were $5 \times 10^{-3} \mathrm{M}, 4 \times 10^{-7} \mathrm{M}$ and $7 \times$ $10^{-7} \mathrm{M}$, respectively. The relative standard deviation was less than $9 \%(n=5)$.

\section{Application}

The proposed method was examined with regard to applicability for the determination of tranexamic acid and vitamin $U$ chloride in medicines. The medicines used were five kinds of the commercially available medicine. Three in these medicines were the medicine for stomach and bowels, which contained vitamin $U$ chloride. Others were the medicine for throat and for a cold remedy, which contained tranexamic acid. The shapes of the medicines were granule, capsule and tablet. A liquid toothpaste was also used as a sample which contained tranexamic acid. The samples were made as follows: granule $(1.3-1.5 \mathrm{~g})$ and tablet $(0.3-0.4 \mathrm{~g})$ were dissolved with distilled water and then the solutions were filtered through filter-paper into a 100 or 200-ml calibrated flask. For the capsule-shaped medicine, it was dissolved with distilled water after medicine was taken out from the capsule and weighed precisely. A 20 or $40-\mu l$ portion of the sample solution was further diluted to $10 \mathrm{ml}$ with distilled water. The sample solution made as mentioned above was used to determine tranexamic acid and vitamin U chloride. CL measurement was done in accordance with the procedure described above. The analytical results and the recovery tests were shown in Table 1 and 2 . The recoveries of the tranexamic acid and vitamin $U$ chloride were 96 $103 \%$. The concentration of tranexamic acid and vitamin $U$ chloride in the drugs except for $C$ sample corresponded to the concentration labeled. These results were satisfactory. It was concluded that this method can be applied to the other real samples.

\section{References}

1. K. Sato and S. Tanaka, Microchem. J., 53, 93(1996).

2. T. Fujimaki, T. Tani and S. Watanabe, Anal. Chim. Acta, 282, 175(1993).

3. K. Sato, Y. Tobita and S. Tanaka, Bunseki Kagaku, 44, 765(1995).

4. N. Hanaoka and H. Tanaka, J. Chromatogr., 606, 129 (1992).

5. P. J. M. Kwakman, H. Koelewijn, I. Kool, U. A. Th. Brinkman and G. J. \& Jong, J. Chromatogr., 511 , 155(1990).

6. R. L. Heirikson and S. C. Meredith, Anal. Biochem., 136, 65(1984).

7. M. Roth, Anal. Chem., 43, 880(1971).

8. E. Bayer, E. Grom, B. Kaltennegger and R. Uhmann, Anal. Chem., 48, 1106(1976). 